\title{
TRES NOVAS TORNARIAS (Enteropneusta) DAS COSTAS SUL DO BRASIL
}

Tagea K. S. Björnberg

Do Museu Paranaense e Faculdade de Filosofia, Ciências e Letras da Universidade do Paraná

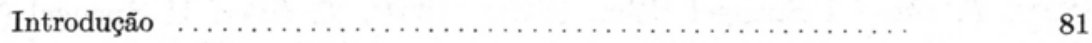

I. Tornaria mourei n. sp. ........................ 82

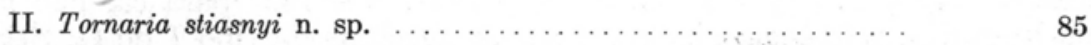

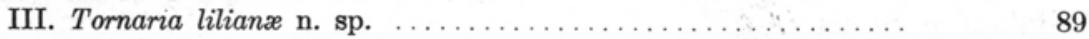

Conclusões gerais : correspondência das formas larvais $\ldots \ldots \ldots .92$

Correlação dos têrmos técnicos . . . . . . . . . . . . . . . . 93

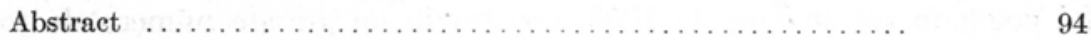

\section{INTRODUÇÃO}

Foram-me cedidas para estudo, pelo Instituto Oceanográfico, da Universidade de S. Paulo, várias larvas de vermes Enteropneusta helminthomorpha, encontradas no plancton colhido atrás da Ilha do Bom Abrigo e ao largo de Cananéia, no Estado de S. Paulo.

Não há na literatura sôbre o grupo dos Enteropneusta referência alguma à descrição de tornárias brasileiras. Fr. Müller foi o único a deixar notas publicadas em 1889 sôbre a existência de tornárias nas costas do Brasil, não chegando a descrevê-las. Segue-se o que escreveu a respeito : "Já desde 1860 eu conheço larvas (Tornárias) que indubitavelmente pertencem ao mesmo gênero (Balanoglossus), não obstante naquele tempo passarem geralmente por larvas de estrelas do mar. Resta indagar se são da mesma espécie (B. gigas)". Mais adiante, no mesmo artigo (1898): "Nos mêses de fevereiro e março tinha eu encontrado, há mais de vinte anos, no mar que banha a praia de fóra da capital da província (de Sta. Catarina), larvas de alguns Balanoglossus (Tornárias) que naquele tempo ainda passavam por larvas de alguma estrela do mar. Era pois de presumir que pelo fim de janeiro e nas primeiras semanas de fevereiro aparecessem os ovos e os primeiros estados larvais, e se assim fosse, deviam ser superabundantes em uma localidade (Armação de Piedade) onde tão frequentemente se encontram os animais adultos, visto como os ovos produzidos por uma única fêmea devem contar muitas centenas de milhares". Segundo Spengel, Fr. Müller chega mesmo a informar por carta a Keferstein sôbre a descoberta de um coração pulsátil na tornária, mas, não a descreve (seg. 
citação de G. Stiasny-Wijnhoff e G. Stiasny - 1931, p. 66). É pena que Fr. Müller não se tenha dado ao trabalho de, pelo menos, fazer um desenho da tornária que descobriu.

As larvas fornecidas pelo Instituto Oceanográfico são todas do tipo I de Stiasny-Wijnhoff e Stiasny (1931, p. 185-186) pois "possuem um campo pré-oral com a forma de âncora" e são "tornárias sem tentáculos" caracterizadas pela forma de duplo cône, tamanho médio, lingueta ventral normal, falta de lobos ventrais inferiores, presença de celoma situado peri-intestinalmente, intestino médio grande, entumescido". As tornárias já descritas, que pertencem a esse tipo, são as' seguintes: de Balanoglossus clavigerus (G. Stiasny, 1914), Tornaria bournei (G. C. Bourne, 1889), T. krohni (A. Krohn, 1854), T. mülleri (John Müller, 1848), T. weldoni (T. H. Morgan, 1894), T. ritteri I Spengel (Wm. Ritter, 1894), T. ritteri II Spengel (Wm. Ritter e B. M. Davis 1904), T. meeki (G. Stiasny 1925), T. mielcki G. Stiasny 1926, T. tergestina G. Stiasny 1927. De 1927 para cá, não houve na literatura referência alguma a novas tornárias do tipo I de G. StiasnyWijnhoff e Stiasny.

A profundidade da proveniência das amostras a mim fornecidas é de $10 \mathrm{~m}$., aproximadamente. O material oriundo da Ilha do Bom Abrigo foi coletado em janeiro de 1953, constando de grande número de formas jovens e de algumas larvas em estádio máximo de desenvolvimento (estádio Krohn) sendo estas últimas pertencentes a um só tipo de tornária, enquanto que as jovens quase todas a outro tipo. As larvas provenientes de Cananéia foram coletadas em 28 de setembro de 1949 e estavam quase todas no estádio Spengel de desenvolvimento, algumas no estádio Agassiz e uma no estádio Krohn, pertencendo quase todas a um ou outro dos tipos jovens das da Ilha do Bom Abrigo. Todo o material foi fixado em formol a $4 \%$ e assim por mim recebido. Ignoro o grau de contração das tornárias.

\section{Classe ENTEROPNEUSTA Gegenbaur 1870}

I. Tornaria mourei n. sp.

Est. I - Figs. 1-8

Tipo - No Museu do Instituto Oceanográfico, da Universidade de S. Paulo.

Tоро-тіро - Museu Paranaense.

OcorrêncIa - Ilha do Bom Abrigo.

Data dA COleta - Janeiro de 1953.

Diagnose - Idade, estádio Krohn de desenvolvimento progressivo (Est. I - Figs. 1-3). Tamanho: de 1,2 a $1,3 \mathrm{~mm}$ de altura, por $1,1 \mathrm{~mm}$ de largura. Forma, de duplo cône, sendo a área anal bem abaulada (Est. I - Fig. 1). Placa apical situada na região média ou ligeiramente dorsal (Est. I - Fig. 3). Faixa ventral não muito estreita nem muito larga, em 
posição quase horizontal lateralmente (Est. I - Figs. 1 e 2). Ārea pré-oral, mais ou menos do mesmo tamanho ou ligeiramente menor (Est. I - Fig. 7) que a post-oral. Forma da área pré-oral, em âncora e convexa, sendo o lábio superior de grande concavidade e sobressaindo um pouco (Est. I Figs. 1 e 7). Os dois celomas do tronco e colar acham-se presentes no estádio Krohn (Est. I - Fig. 1), portanto bastante precoces em aparecimento. Esta larva não apresenta um anel anal (Est. I - Figs. 1 e 3). Anel ciliado circular inferior, ligeiramente ondulado (Est. I - Figs. 1 e 3).

Caractéres específicos desta tornária: Forma da lingueta ventral, bem alta e estreita (Est. I - Figs. 1 e 6) e em uma das tornárias apresenta um largo lobo de cada lado (talvez efeito de contração. Est. I - Fig. 1). Reentrância bucal, alta e pequena (Est. I - Fig. 2). Lábios superior e inferior, paralelos (Est. I — Fig. 1).

Ārea oral, mais ou menos estreita (Est. I - Figs. 2, 3, 5, 6 e 7). Linguetas secundárias, muito salientes ; largas na base e terminando quase em ponta. Existem duas na face interna de cada lingueta primária e 2 a 3 na face externa das mesmas, sendo as superiores menos salientes (Est. I - Figs. 1, 2 e 7) e a mais inferior, mais saliente que todas as outras (Est. I - Fig. 7). Na lingueta média da área pré-oral há 4 linguetas secundárias, sendo as 2 medianas bem definidas e as dos extremos apenas esboçadas (Est. I - Figs. 1 e 7). Tanto a lingueta média pré-oral como a post-oral, mais altas e largas que as laterais correspondentes (Est. I Fig. 7).

Lobos primários dorsais inferiores, profundos (Est. I - Figs. 3-5 e 7). Terminam voltados para cima, mais ou menos em ponta. Os lobos secundários, na maioria profundos e, às vezes, estreitos, abrindo-se em ângulos quase retos (Est. I - Figs. 7, 1-3).

Lobo lateral, bem acentuado, situado mais ventralmente que lateralmente (Est. I - Figs. 7, 1 e 2). Forma do lobo lateral, arredondada, em garrafão, limitado ventralmente por uma lingueta alta e larga e dorsalmente por uma lingueta menor, mas, também arredondada (Est. I - Figs. 7 e 2).

Não há aparentemente indícios de aberturas branquiais no estádio Krohn. O intestino médio, cilíndrico e entumescido (Est. I - Fig. 3). O hidrocela é bem desenvolvido no estádio Krohn, apresentando já engrossamento muscular das paredes (Est. I - Figs. 2 e 3). A vesícula sanguínea da proboscis já se delineia (Est. I - Fig. 2), o pericárdio acha-se presente no vão entre o canal de escoamento do hidrocela e a vesícula sanguínea da proboscis (Est. I - Fig. 2). O lúmen do hidrocela, grande, sendo ele em forma de pêra ou fuso (quando visto lateralmente) (Est. I - Figs. 2 e 3). $\mathrm{O}$ cordão que liga o hidrocela à placa apical, longo e fino (Est. I - Figs. 4 e 5). Hidróporo situado mais ou menos na linha mediana e um pouco à esquerda (Est. I - Fig. 4).

Discussão - As figuras 4 a 6 mostram a mesma tornária em estado de desenvolvimento regressivo, isto é, no estádio Spengel, pois de acôrdo 
com G. Stiasny-Wijnhoff e G. Stiasny (1927, p. 44), o estádio Spen: se reconhece pela "involução dos lobos e linguetas secundárias, celom do tronco e do colar presentes, tamanho menor que no estádio preceder (Krohn), forma de clava, acinturamento no meio do corpo, em forma anel, menos transparente".

Confronto dos caractéres das tornárias até hoje descritas, diferentes , Tornaria mourei $n$. $s p$.

Tornària de Balanoglossus clavigerus - Tamanho: $2 \mathrm{~mm}$. Hidrc cela abrindo-se por um poro mediano ; o lábio superior da área oral pouc côncavo ; anel ciliado anal ; o anel ciliado circular inferior não ondulado lingueta ventral arredondada e larga; linguetas secundárias largas.

Tornaria bournei (descrita por G.C. Bourne 1889 ; por G. Stiasn: 1921) - Hidrocela, só aparece no estádio Spengel ; intestino médio en forma de balão, redondo ; placa apical, média ; área pré-oral, empurradi para perto da placa apical; celomas de aparecimento tardio; linguet: ventral baixa e larga ; área oral muito larga ; lobos primários dorsais infe riores terminados em ponta; lingueta que limita o lobo lateral do lade dorsal quase inconspicua.

Tornaria mülleri (descrita por Müller 1848 ; por G. Stiasny 1921) Tamanho: $1 / 2 \mathrm{~mm}$. Intestino médio em balão ; área anal semi-esférica; lábio superior da área oral muito pouco côncavo ; celomas ainda não desenvolvidos no estádio Krohn ; lingueta ventral mais larga que alta ; reentrância bucal no meio do corpo ; área oral muito larga ; linguetas secundárias muito pouco salientes; lobos primários dorsais inferiores muito pouco profundos; lobos secundários pouco salientes, em lugar do lobo lateral, uma lingueta.

Tornaria krohni (Krohn 1854) - Tamanho : 2 mm. Hidróporo um pouco à direita; intestino médio em balão, pequeno; área anal semi-esférica; área oral, pequena ; lingueta ventral, não paralela ao lábio superior ; área oral larga ; linguetas secundárias muitíssimo salientes e largas, 4-5 linguetas secundárias nas linguetas primárias laterais das áreas pré e post-orais ; lingueta média da área pré-oral mais larga e mais baixa do que as laterais, formato do lobo lateral.

Tornaria meeki (descrita por Meek 1922 e por Stiasny 1925) Tamanho: 1,5 a $1,75 \mathrm{~mm}$. Concavidade inferior da área pré-oral pequena; apenas o celoma do tronco presente no estádio Krohn; área oral muito larga, forma de lobo lateral.

Tornaria mielcki G. Stiasny 1926 - Tamanho : 1,5 a $2 \mathrm{~mm}$. Intestino médio grande, redondo ; largura enorme da faixa ciliada circular inferior $(2,25$ a $2,5 \mathrm{~mm})$, em aba de chapéu; apenas celoma do tronco no estádio Krohn.

Tornaria weldoni (descrita por T. H. Morgan 1894 e por G. Stiasny 1920) - Tamanho: $2,5 \mathrm{~mm}$. Presente no estádio Krohn apenas o celoma 
do tronco ; linguetas secundárias muito salientes mas, estreitíssimas, 4 a 6 em cada lingueta primária.

Tornaria ritteri I Spengel (descrita por Ritter 1894) e Tornaria ritteri II Spengel (descrita por Wm. E. Ritter e B. M. Davis 1904). Tamanho : 2,07 e 1,9 mm. Årea pré-oral larga ; celomas só aparecem antes da metamorfose ; lingueta ventral larga e baixa ; área oral larga horizontalmente ; linguetas secundárias curtas e grossas, formas dos lobos laterais, diferentes.

Tornaria tergestina G. Stiasny 1927 - Tamanho : 0,5 a $0,6 \mathrm{~mm}$. Hidrocela estreito, tubular; intestino médio esférico; área anal quase chata ; concavidade da área oral muito pequena ; apenas celoma do tronco no estádio Krohn ; lingueta ventral muito baixa e larga ; área oral muito larga; lobos primários dorsais inferiores não muito profundos, lobos secundários largos, lobo lateral raso.

A tornária da Ilha do Bom Abrigo não concorda portanto com a descrição de nenhuma das tornárias já descritas e pertencentes ao tipo I. Considero-a, pois, como espécie nova de tornária, passando ela a denominar-se Tornaria mourei $\mathrm{n}$. sp., até ser descoberta a forma adulta a qual dá origem. Veja-se no fim do trabalho a discussão sôbre êsse assunto.

\section{Tornaria stiasnyi n. sp.}

Est. I - Fig. 8; Est. II - Figs. 9-21

Tipo - No Museu do Instituto Oceanográfico

Topo-tipos - No Museu Paranaense

OCorrência - Cananéia e Ilha do Bom Abrigo

DAta - Estádios jovens, janeiro; estádios adiantados, setembro.

Diagnose - Tamanho : de 1,1 a $0,8 \mathrm{~mm}$ de altura, por 0,8 a $0,6 \mathrm{~mm}$ de largura. Idade das larvas coletadas em Cananéia: estádios Spengel e Agassiz (Est. I - Fig. 8 ; Est. II - Figs. 9 - 13). Forma : duplo cône ; área anal, extremamente elevada na região ventral, pouco elevada ou achatada na região dorsal. Placa apical, bem dorsalmente situada (Est. II - Fig. 12) e bem delimitada. Faixa ventral relativamente estreita, na região ventral, em posição horizontal, dorsalmente vertical. Ārea pré-oral ocupá menos da metade da região ventral, é mais apical que ventral, com a forma de âncora. Lábio dorsal da área oral extremamente côncavo (Est. I - Fig. 10). Celomas do tronco e colar, presentes no estádio Spengel. Anel ciliado circular inferior apresenta-se com reentrâncias e saliências em bico, sendo os cílios que o adornam excepcionalmente longos. Acima do mesmo, na faixa ventral, encontramos uma série de regiões salientes, rugosas, das quais chegamos a contar $12 \mathrm{em}$ um espécime. Nas maiores saliências das linguetas laterais das áreas pré e post-orais, também existem destas manchas, uma em cada saliência. Futuramente serão feitos cortes histológicos para a determinação da natureza dessas manchas. 
Anel anal, ausente. Anus excêntrico. Um pequeno círculo ao redor do anus se apresenta ligeiramente rugoso (Est. II - Fig. 12), ao contrário do que sucede no resto da região anal que é lisa, exceto na região dorsal onde há duas manchas de pele rugosa simétricamente situadas, lobo acima, no anus (Est. I - Fig. 11). Lingueta ventral alta e estreita (Est. I - Fig. 8 ; Est. II - Figs. 12, 16, 18). Os dois lábios da área oral, mais ou menos paralelos (Est. II - Figs. 19-21, 14). Ārea oral, larga (Est. I - Fig. 8 ; Est. II - Figs. 12, 14, 21). Lobos dorsais inferiores profundos e voltados para cima, terminando em ponta de dedo. Lobo lateral, muito profundo e grande (Est. I - Fig. 8 ; Est. II - Figs. 12, 13). Lingueta ventral do lobo lateral, muito grande. Lingueta dorsal, do lobo lateral, pequena e mais estreita que a ventral. Hidrocela, esférico, de lúmen grande (Est. I - Figs. $8,11)$; quando visto lateralmente, tem a forma de pêra. Hidróporo, esquerdo. $\mathrm{O}$ cordão que liga o hidrocela à placa apical, é longo e estreito. Visto de cima e mesmo de lado, no estádio Spengel (Est. II - Figs. 21 e 23), o hidrocela mostra-se bem próximo do intestino, mas não encostado nêle, tendo apenas as duas extremidades ou paredes laterais tocando a superfície do mesmo. Inferiormente, onde começa o canal de escoamento, acha-se o pericárdio e o espaço que será o futuro seio sanguíneo. Intestino, o esôfago é curto e ligeiramente curvo. Intestino médio, cilíndrico e oval. Intestino final, grande e em forma de cône invertido. Há um diafragma separando cada intestino do outro (Est. II - Fig. 12 ; Est. I - Fig. 11). No estádio Spengel, já parece estar esboçada uma brânquia. Nota-se, pelo menos, um grande engrossamento muscular da parte posterior do esôfago, espessamento êsse que se acentúa no estádio Agassiz (Est. I Fig. 8 ; Est. II - Fig. 13). Celomas, do colar e do tronco, muito estreitos e em forma de salsicha, sendo o do tronco maior e o do colar apenas esboçado no estádio Spengel, terminando este em ponta. No estádio Agassiz, já se encontra mais desenvolvido (Est. I e II - Figs. 8, 10 - 13).

Discussão - Em janeiro de 1953, foram recolhidas, próximo à Ilha do Bom Abrigo, formas jovens de tornárias que foram consideradas como sendo a esta pertencentes, por apresentarem as mesmas características (as acima grifadas) das tornárias descritas acima (veja Est. II - Figs. 14 a 21), isto é, área anal extremamente elevada na região ventral e pouco elevada ou quase chata na dorsal ; área pré-oral empurrada para a placa apical ; placa apical dorsalmente situada; anus excêntrico ; manchas de pele rugosa nos mesmos pontos onde foram encontrados acima; anel anal ausente; área oral muito larga; reentrância bucal muito elevada; forma dos três intestinos idêntica. As Figs. 15, 16 e 17 da Est. II mostram, respectivamente, o aspecto ventral, lateral e dorsal de uma fase de transição de Heider para o estádio Metchnikoff jovem do desenvolvimento progressivo.

O estádio de Heider, de acôrdo com Stiasny-Wijnhoff e Stiasny (1931, p. 44) é caracterizado por "faixa ciliada longitudinal sem presença de lobos e linguetas primários, faixa ciliada circular acabando de se formar" e o estádio Metchnikoff, também do desenvolvimento progressivo da tornária, deve apresentar "lobos primários e linguetas em desenvolvimento ou já 
formados ; celoma do tronco ainda não presente ou acabando de aparecer" (autores citados 1931, p. 44). Como se vê pelas figuras, os lobos e linguetas primárias estão em formação muito incipiente, os celomas do tronco ainda nem esboçados, a tornária portanto em fase de transição entre o estádio Heider e Metchnikoff ou num estádio Metchnikoff muito jovem. O tamanho destas tornárias é de $0,34 \times 0,30 \mathrm{~mm}$. A Fig. 18 mostra uma fase um pouco mais adiantada do que esta (tamanho: $0,51 \times 0,35 \mathrm{~mm}$ ), vista de perfil. Note-se a elevada situação da reentrância bucal já nessa fase jovem. As Figs. 19, 20 e 21 mostram uma fase Metchnikoff jovem de desenvolvimento progressivo da larva vista de dorso, de ventre e de perfil, assim como as da área post-oral. Os celomas ainda não se esboçaram. Já se notam os cílios em formação na faixa ciliada circular inferior, que já começa a elevar-se. A lingueta ventral é muito elevada desde o estádio Metchnikoff jovem anterior. A Fig. 14 nos mostra a mesma larva em estádio Metchnikoff adiantado, com começo de esboço de lobos secundários, inclusive aparecimento da lingueta ciliada inferior. De acôrdo com StiasnyWijnhoff e Stiasny (1931, p. 44) o estádio Krohn de desenvolvimento progressivo da larva se caracteriza por "linguetas e lobos secundários ou tentáculos respectivos em formação ou já formados ; celoma do tronco em geral presente ; celoma do colar ainda não ou apenas esboçado; maior altura, ponto culminante do desenvolvimento larvário" (grifo do autor)). Pelos caractéres apresentados, a larva da Fig. 14 deveria ser considerada como um estádio Krohn jovem, prefiro porém considerá-la como ainda um estádio Metchnikoff adiantado, em virtude do seu tamanho que é de, apenas, 0,56 x $0,30 \mathrm{~mm}$, muito pequeno para ser de um estádio Krohn de larva cujos estádios Spengel e Agassiz apresentam 1,1 a $0,8 \mathrm{~mm}$ de altura, por 0,8 a $0,6 \mathrm{~mm}$ de largura. O estádio Krohn seria, nesta larva, no mínimo de 1,1 $\mathrm{mm}$ de altura por $0,8 \mathrm{~mm}$ de largura e, provavelmente, (de acôrdo com o autor acima citado), maior. Embora neste estádio seja considerado por StiasnyWijnhoff e Stiasny (1931, p. 50) como a condição sem a qual não é possível classificar uma tornária ("trata-se em cada tornária de conhecer o estádio Krohn, o ponto culminante do desenvolvimento larvário" quando "aparecem todos os caractéres larvais".), creio que esta difere tanto pela morfologia das outras tornárias, todas do tipo I de Stiasny, que não haverá por enquanto possibilidade de confusão com outras formas.

Esta larva parece-se muito com a Tornaria hubbardi Ritter e Davis 1904, com a qual tem vários caractéres em comum : forma de sino, anus excêntrico, ondulação do anel ciliado circular inferior, grande comprimento dos ćlios, área anal muito achatada e intestino inteiro deslocado para a região anal. Os próprios Stiasny-Wijnhoff e Stiasny classificaram-na à parte das tornárias do tipo I, escrevendo "nestes 4 grupos I, II, III, IV, que representam 4 tipos diferentes, é possível classificar todas as tornárias até agora conhecidas com exceção de T. hubbardi que, por isso, constituiria um grupo próprio ou tipo V. Ela pertence às tornárias não tentaculadas com área pré-oral em forma de âncora e celomas peri-intestinais, porém difere da maioria dos pertencentes a êste grupo pela forma do corpo, a falta de um lobo lateral e a presença de uma grande lingueta lateral". A tornária 
ora descrita difere de $T$. hubbardi pelo fato de apresentar menor número de aberturas branquiais, uma placa apical bem delimitada (Est. I e II Figs. 8, 12), uma área anal mais protuberante, área oral menos larga, um lobo lateral, áreas pré e post-orais maiores, além da forma que não é de sino pontudo, mas de sino arredondado superiormente.

A comparação entre as tornárias foi feita no mesmo estádio de desenvolvimento, isto é, no estádio de Spengel ou de Agassiz. Para resolver qual dêstes, de acôrdo com Stiasny-Wijnhoff (1931, p. 122 - 123) "a larva aqui representada corresponde talvez ao estádio Agassiz do B. clavigerus apesar da área anal não ser protuberante, porém completamente achatada. Sete aberturas branquiais, divertículo endodérmico, celomas do tronco e colar já desenvolvidos, as faixas ciliadas longitudinais pouco onduladas que ficam em forma de capa sôbre a região apical - todos caractéres que indicam o estádio Agassiz". Porém, à pg. 185 da mesma obra, os autores escrevem: "há, porém, apenas uma única larva deste tipo conhecida, que se encontra em estádio de desenvolvimento adiantado, na qual, em geral, os corpos larvais não apresentam mais a forma de sino, mas, são alongadas, pelo menos possuem uma área anal protuberante". A tornária que estou descrevendo, quando considerada à parte das formas jovens, também daria a impressão de um estádio Agassiz de desenvolvimento, em virtude de possuir : área anal protuberante, em forma de cône, intestino deslocado para trás, para a área anal, enorme hidrocela, faixa ciliada longitudinal empurrada para a placa apical. Quando comparada com formas jovens, porém, observaremos que já no estado de transição entre Heider e Metchnikoff, a larva exibe todas as características mencionadas acima, excetuando o grande hidrocela e, talvez, a área anal proeminente, em forma de cône (observem-se, para tal fim, as Figs. 8 a 11). O último caráter mencionado não parece ser de tal importância para caracterizar o estádio Agassiz, mesmo para o próprio Stiasny, que não pôde observá-lo em T. hubbardi pelo fato desta não possuir área anal protuberante e sim completamente chata. Diante dos estudos feitos com a tornária presentemente descrita, o autor prefere colocar tanto $T$. hubbardi como a larva representada nas Figs. 8-11, no estádio Spengel de desenvolvimento, considerando a forte ondulação da faixa ciliada longitudinal (restos de linguetas e lobos secundários em involução), a forma das larvas ainda não alongadas, a grande transparência, o menor tamanho dos celomas. A Fig. 13 representará, nesse caso, uma larva em estádio Agassiz de desenvolvimento regressivo porque já é nítido o alongamento geral da larva, o aumento considerável em tamanho dos celomas, o maior desenvolvimento muscular da base do esôfago, conjunto hidrocela, pericárdio, seio sanguíneo mais desenvolvido e menor transparência da larva.

As características do estádio Spengel são (Stiasny-Wijnhoff e Stiasny, l. c., p. 44) "lobos e linguetas secundárias em involução ou já involuidas ; celomas do colar e do tronco presentes, tamanho menor que o do estádio precedente; forma de clava, no meio do corpo um acinturamento anelar ; menor transparência”. As Figs. 8-11 da Est. I, parecem satisfazer essas 
exigências, permitindo-me assim a afirmação acima, pelo menos para a tornária em estudo neste trabalho. Como esta difere de T. hubbardi, com a qual mais se parece, de todas as tornárias descritas anteriormente, considero-a como espécie nova de tornária, dando-lhe o nome de Tornaria stiasnyi n. sp., em honra ao maior estudioso de tornárias. Este nome permanecerá até ser descoberta a forma adulta que pertença à tornária.

\section{Tornaria lilianae n. sp.}

Est. II — Figs. 24-30

\section{Tipo - Museu do Instituto Oceanográfico}

Topo-TIPo - Museu Paranaense

Ocorrência - Cananéia e Ilha do Bom Abrigo

Data - Estádio Krohn : 28/9/1949; estádio Metchnikoff : Janeiro

Diagnose - Tamanho: altura, 0,7 $\mathrm{mm}$; largura, 0,6 mm. Forma : de duplo cône, área anal, pouco abaulada ; faixa ventral, em posição vertical (Est. II - Fig. 24), placa apical de situação média e dorsal (Est. II — Fig. 25) ; faixa ventral, estreita. Ārea pré-oral, em forma de âncora (Est. II - Fig. 26), mais ou menos igual, em tamanho, à post-oral (Est. II Fig. 27), convexa, grande, ocupando mais ou menos a metade da região ventral da tornária, não sendo empurrada para o lado apical. Lobo lateral, presente. Situação dos celomas, peri-intestinal (Est. II - Fig. 25).

Anel ciliado inferior, muito conspícuo e duplo, não ondulado, ou com pouquíssima ondulação. Anel anal, presente (Est. II - Figs. 24 - 26).

Forma da lingueta ventral, mais achatada que alta, de largura média (Est. II - Figs. 25 e 26). Reentrância bucal, estreita, ocupando mais ou menos o meio do corpo (Est. II - Fig. 25). Lábio superior, paralelo ao inferior (Est. II - Fig. 26). Área oral, estreita, pequena (Est. II Fig. 25). Linguetas secundárias, pouco salientes, em número de 2-4 nos lobos primários internamente e de 3-4 nos lobos primários externamente (Est. II - Figs. 25, 27, 28). Linguetas médias das áreas pré e post-orais com 3 linguetas secundárias. Lobos primários dorsais inferiores, profundos, terminando perto um do outro e seguindo horizontalmente (Est. II — Fig. 24). Lobos secundários, muito pouco profundos. Lobo lateral, largo e achatado, com uma lingueta pouco saliente, mas bem demarcada do lado ventral e esboço apenas de lingueta, dorsalmente (Est. II - Figs. 25 e 27). Idade dos celomas, presentes no estádio Krohn. Pericárdio, notocórdio e brânquias ainda não presentes no estádio Krohn. Poro do hidrocela, abrindo-se um pouco à esquerda (Est. II — Fig. 24). Hidrocela, mais ou menos em forma de pêra, alongado, quando visto lateralmente e redondo, quando em plano ventral. Não apresenta esporas. Lúmen grande. Cordão que a liga à placa apical, longo e estreito. $\mathrm{O}$ canal de escoamento do hidrocela, não distintamente separado dela e curto. Posição do hidrocela : entre o esôfago e o estômago, sôbre os quais está assentado. 
Intestino, dividido nitidamente em três partes (Est. II - Fig. 24). Intestino médio, cilíndrico e acinturado ; intestino final, cônico e curto. Esôfago, das três tornárias descritas, é o mais longo e curvo, porque a bôca acha-se situada mais inferiormente que nas outras tornárias (Est. II Fig. 25). Celomas do tronco, com a borda inferior assentada na parte mais larga inferior do intestino médio (Est. II - Fig. 26). Os celomas, ambos lameliformes, mais largos que celomas de $T$. stiasnyi e não retangulares como os de T. mourei (Est. II - Fig. 25). Celoma do colar, bem mais perto, não encostado ao intestino médio (Fig. 24). Acha-se localizado logo acima do celoma do tronco. Esta tornária é pouco transparente.

\section{DISCUSSÃO, DE Tornaria lilianae, n. sp. E CONFRONTO COM OS CARACTERES DISCORDANTES DE OUTRAS TORNARIAS}

Tornária de $\boldsymbol{B}$. clavigerus d. Chiaje - Tamanho: maior; poro do hidrocela, mediano; hidrocela, maior ; intestino médio, não apresenta acinturamento ; linguetas secundárias, são salientes, em número de 3-4; lobos primários dorsais inferiores, terminados voltados para cima; lobos secundários, profundos em estado adiantado; lobo lateral, profundo.

Tornaria bournei - Intestino médio, em balão, tamanho maior ; placa apical, de situação média ; celomas, não presentes no estádio Krohn ; anel ciliado inferior, ondulado; 3-4 linguetas secundárias; lobos secundários estreitos; lobo lateral profundo.

Tornaria muilleri - Intestino médio, em balão, grande ; área anal, semi-esférica ; celomas no estádio Krohn, não presentes; anel ciliado circular inferior, ondulado ; área oral muito larga ; lobos primários dorsais inferiores, inclinados para cima; não existe lobo lateral.

Tornaria krohni - Tamanho maior ; área anal semi-esférica ; intestino médio em balão; placa apical ventralmente situada; celoma do tronco apenas presente no estádio Krohn ; lingueta ventral alta e estreita ; lábio superior não paralelo ao inferior ; área oral muito larga ; 4 - 5 linguetas secundárias; lobos primários dorsais inferiores, voltados para cima; lobos secundários, estreitos e profundos; lobo lateral de formato irregular e profundo.

Tornaria meeki - Tamanho maior ; apenas celoma do tronco presente no estádio Krohn ; anel ciliado circular inferior, ondulado ; lingueta ventral muito alta e estreita; reentrância bucal estreita ; área oral muito larga ; 3 linguetas secundárias ; lobos primários dorsais terminam voltados para cima.

Tornaria mielki - Tamanho muito maior ; intestino médio, redondo ; faixa ventral larga e horizontal ; apenas celoma do tronco presente no estádio Krohn; lingueta ventral, alta e estreita ; reentrância bucal, alta e estreita ; linguetas secundárias, salientes ; 4 linguetas secundárias ; lobos secundários, profundos; lobo lateral muito profundo. 
Tornaria weldoni - Tamanho muito maior ; área oral pequena ; apenas celoma do tronco presente no estádio Krohn; lingueta ventral alta e estreita ; reentrância bucal muito alta e estreita ; linguetas secundárias muito salientes e estreitas, em número de 4-6; lobos secundários, muito profundos e estreitos; lobo lateral muito profundo.

Tornaria ritteri - Tamanho muito maior ; área anal muito abaulada; faixa ventral assaz larga; celomas de aparecimento tardío ; área oral muito larga, horizontalmente ; lobo lateral, tanto de T. ritteri I, como de $T$. ritteri II, muito profundos.

Tornaria mourei - Tamanho maior ; área anal bem abaulada ; anel ciliado inferior, ondulado ; lingueta ventral alta e estreita ; abertura bucal, alta ; esôfago mais curto ; hidrocela, maior ; intestino médio não acinturado; lobos secundários mais profundos e voltados para cima; lobo lateral, profundo; forma dos celomas diferente.

Tornaria stiasnyi - Área anal diferente; situação diferente da placa apical ; tamanho e posição da área pré-oral ; ondulação grande do anel ciliado circular inferior ; altura da reentrância bucal ; área oral larga ; presença das manchas de pele rugosa ; lobos primários dorsais inferiores, profundos; lobo lateral, profundo e largo ; comprimento do esôfago e forma dos celomas.

Tornaria tergestina - Intestino médio esférico; área oral muito larga; área pré-dorsal muito pequena; apenas celomas do tronco presentes no estádio Krohn; reentrância bucal, alta e estreita ; linguetas secundárias, salientes, em número de $3-4$; lobos dorsais inferiores não muito profundos; lobos secundários profundos e largos.

Constatando-se, por todas essas observações, a não coincidência da descrição desta tornária com a de nenhuma outra, foi-lhe atribuido o nome de Tornaria lilianae, até que seja descoberto o Enteropneusta adulto do qual se origina.

A descrição e a diagnose acima se referem ao estádio Krohn da tornária descoberta entre os vários exemplares de Tornaria stiasnyi n. sp., coletados no plancton de Cananéia, em setembro de 1949. Havia, apenas, um exemplar da mesma. As linguetas e lobos secundários estão pouco acentuados, a tornária é pouco transparente, o lobo lateral pouco profundo, o que leva a crer que o estádio Krohn, nesta tornária, já está terminado. Não é ainda estádio Spengel pelo fato de não apresentar um acinturamento mediano. As Figs. 29 e 30 mostram a mesma tornária em estádio Metchnikoff jovem de desenvolvimento progressivo (tamanho menor $=0,39 \mathrm{x}$ $0,27 \mathrm{~mm}$ ). Estas formas foram apanhadas no plancton coletado próximo da Ilha do Bom Abrigo, em janeiro deste ano. Das três novas espécies de larvas, está é a menor e de formato mais regular, além de ser a menos transparente. Mesmo no estádio Metchnikoff, mostra-se menos transparente que as outras tornárias. A posição da bôca, a forma do intestino médio, a área anal de forma regular, o intestino final de mesma forma, o anel anal bem nítido nas formas jovens, a posição vertical da faixa ventral, 
a não ondulação do anel ciliado inferior, permitem facilmənte identificar as formas jovens e adiantadas de Cananéia.

\section{CONCLUSÕES GERAIS - CORRESPONDENCIA DAS LARVAS COM FORMAS ADULTAS}

De acôrdo com G. Stiasny-Wijnhoff e G. Stiasny (1931, p. 188) "Todas as larvas do tipo I caem pois dentro do âmbito de distribuição dos gêneros Balanoglossus e Glossobalanus" e segundo as tabelas publicadas nas páginas 191 e 192 da mesma obra, as tornárias do tipo I pertencem portanto provàvelmente a estes gêneros. Com certeza, determinou-se, apenas, a correspondência entre a larva e a forma adulta de $B$. clavigerus, dentre as diversas larvas do tipo I. As outras correspondências entre larva e forma adulta foram feitas levando apenas em consideração a distribuição geográfica das formas adultas e das larvas.

Se observarmos igualmente no Brasil a distribuição geográfica das formas adultas de Enteropneusta, verificaremos que ocorrem no Rio de Janeiro (Spengel 1893, p. 126) as espécies Glossobalanus minutus (Kowalevsky), Schizocardium brasiliense Spengel, Balanoglossus apertus (Spengel). No Estado de São Paulo, ocorrem Balanoglossus gigas Fr. Müller (Sawaya 1951, p. 730) e Schizocardium brasiliense Spengel (redescoberto pelo Dr. E. Nonato), em São Sebastião ; uma forma menor de Balanoglossus, em Santos. No Estado do Paraná, em Caiobá, ocorre Balanoglossus paranaensis Björnberg e na Ilha do Mel, outra forma parecida de Balanoglossus (Björnberg 1952, p. 367). Em Santa Catarina, ocorrem uma forma pequena de Balanoglossus e uma forma grande, Balanoglossus gigas $\mathrm{Fr}$. Müller (Spengel 1893 , p. 159).

Vemos pois que predominam na costa sul do Brasil as formas pertencentes ao gênero Balanoglossus. As larvas presentemente descritas foram colhidas em zona compreendida entre o litoral paranaense e o litoral de Santos, entre dois locais onde ocorrem formas menores de Balanoglossus. $T$. mourei e T. lilianae são larvas tipicamente pertencentes ao tipo I de Stiasny-Wijnhoff e Stiasny, portanto, provàvelmente, larvas de Balanoglossus, uma delas talvez pertencente a $B$. paranaensis. De acôrdo com Stiasny-Wijnhoff e Stiasny (p. 166 - Tabela geral B das tornárias) todas as tornárias do tipo I, pertencentes a Glossobalanus são em geral menores que as pertencentes a Balanoglossus. Poder-se-ia, por isso, aventar a hipótese de que $T$. lilianae seja larva de um Glossobalanus (talvês G. minutus*) e que T. mourei seja a larva do Balanoglossus paranaensis ou de forma parecida (Veja mapa). Tornaria stiasnyi é uma larva parecidíssima com T. hubbardi, portanto afastada das larvas do tipo I. Para T. hubbardi Stiasny-Wijnhoff e Stiasny (1. c., p. 185) criaram o tipo V, afirmando

* - "O desenvolvimento direto também foi observado em Gloss. minutus (Dawydoff 1927, 1944) mas, em condições anormais. Ordinariamente, este Balanoglosso tem uma larva Tornária”. (Nota de roda-pé da pg. 435 - Dawydoff 1948). 
logo a seguir : "Para os 4 tipos (I, II, III, IV) seguros de tornárias, pudemos estabelecer, com relativa probabilidade, a correspondência. Apenas o quinto tipo apresenta dificuldades. Do mesmo, existe apenas uma única larva, T. hubbardi, da California, mas conhecida. Daí não poder ser dada, presentemente, uma descrição mais detalhada deste tipo. (Ela não é tentaculada, possue forma de sino, possue área pré-oral em âncora e celomas peri-intestinais e, em vez de lobo lateral, uma lingueta lateral). Ao Balanoglossus occidentalis, única forma à qual poderia corresponder, ela não pode pertencer porque nessa região de distribuição foi indicada uma larva do tipo I e aliás, Tornaria ritteri que provàvelmente pertence ao $\mathrm{B}$. occidentalis. Portanto, só seria levado em consideração para correspondência nesse caso o gênero Schizocardium, sôbre cujo desenvolvimento nada se conhece". "A distribuição deste gênero compreende o Perú e o Rio de Janeiro; outros locais não são conhecidos. Uma distribuição de espécies sulamericanas até a Califórnia não é nada rara, de modo que sempre continúa a possibilidade que T. hubbardi esteja em relação com Schizocardium", (Stiasny-Wijnhoff e Stiasny l.c., p. 190-191). A descoberta de T. stiasnyi do mesmo tipo que T. hubbardi, próxima de um local onde ocorre Schizocardium (Canal de São Sebastião) vem apoiar a hipótese do casal Stiasny, sem entretanto confirmá-lo, pois, para isso, seria necessário criar um Schizocardium desde o estado de ôvo e verificar, em primeiro lugar, se o animal não tem desenvolvimento direto, hipótese que não está fora de cogitação. De qualquer modo, $T$. stiasnyi vem contribuir para uma caracterização maior do tipo V, embora ainda não perfeita, dada a falta do estádio Krohn, indispensável para tal. Características deste tipo $\mathrm{V}$ seriam tornárias não tentaculadas, em forma de sino, área pré-oral em âncora e celomas periintestinais, área anal muito elevada anteriormente, grande lingueta lateral na faixa ventral, limitando ou não um lobo lateral, anus excêntrico. A ausência de lobo lateral não seria característica do tipo $V$, uma vez que T. stiasnyi o possúe. Ficam, assim, abertos para a pesquisa vários problemas interessantes com relação às tornárias brasileiras e aos nossos Enteropneusta.

\section{CORRELAÇÃO DOS TERMOS TECNICOS USADOS}

Anel anal $=$ Analring (Stiasny)

Anel ciliado circular inferior = Zirkulärer Wimperring (Wimperkranz) (Stiasny), circum-anal ciliated band (Bourne), peri-anal ring of cilia (Balfour), circular band (Tokioka). Área anal $=$ Analfeld (Stiasny).

Área oral $=$ Oralfeld $($ Stiasny), middle lateral area + anterior \& posterior lateral area or fold (Morgan).

Área pré-oral = Präoral feld (Stiasny), preoral ring (Morgan).

Área post-oral = Postoral feld (Stiasny), postoral ring (Morgan). sac (Morgan).

Coração (pericárdio) $=$ Herzblase (Pericardium) (Stiasny), heart or proboscis

Faixa ciliada longitudinal $=$ longitudinal Wimperschnur (Stiasny), longitupinal ciliated band (Balfour). 

(Bourne).

Faixa ciliada longitudinal pré-oral $=$ Præoral schnur (Stiasny), preoral band

Faixa ciliada longitudinal postoral $=$ Postoral Wimperschnur (Stiasny), postoral ring (Balfour).

Faixa ventral $=$ Ventralband (Stiasny).

Hidrocela $=$ Wasserblase (Stiasny), Wassersack (Hydrocœl) (Stiasny); proboscis cavity (Bourne) ; water vascular vesicle (Balfour) ; anterior enterocœl (Morgan) : hydrocœlom (Tokioka).

Hidróporo $=$ Hydroporus (Stiasny) ; proboscis pore (Bourne) ; water pore (Morgan e Tokioka). (Morgan).

Lábio superior $=$ Oberlippe (Stiasny); pre-oral part of longitudinal band (Morgan).

Lábio inferior $=$ Unterelippe (Stiasny) ; postoral part of longitudinal band

Lingueta média da área pré-oral = Mittelstreifen des Präoralfeldes (Stiasny); median loop of pre-oral band (Bourne).

Lingueta média da área post-oral $=$ Mittelstreifen des Postoralfeldes ; median loop of longitudinal band (Bourne). band (Morgan).

Lingueta primária $=$ Primärer Sattel (Stiasny); upper turn of longitudinal

Lingueta secundária $=$ Sekundärer Sattel (Stiasny).

Lingueta ventral $=$ Ventral Sattel (Stiasny); ventral saddle (Tokioka).

Lingueta ventral do lobo lateral = Lateral Sattel (Stiasny); upper wave of lower limb of longitudinal ciliated band (Morgan).

Lingueta lateral $=$ Primärer Sattel (Stiasny); lateral loop (Bourne).

Lobo dorsal inferior $=$ Unterer dorsal Loben (Stiasny)

Lobo lateral $=$ Lateral Lobus (Stiasny).

Lobo primário ventral $=$ Obere primäre ventrale Loben (Stiasny) ; anterior lateral area (fold) (Morgan); upper ventral lobe (Tokioka).

Lobo secundário = Sekundärer Lobus (Stiasny).

Placa apical $=$ Scheitel platte (Apikalplatte) (Stiasny) $;$ apical sense organ (Bourne); pre-oral lobe (Balfour); apical plate (Morgan).

Poro do hidrocela $=$ (veja hidróporo).

\section{ABSTRACT}

The "Instituto Oceanográfico", of the University of S. Paulo, lent me several tornariae for studying purposes. These larvae were obtained from samples of plankton collected off the Island of Bom Abrigo (see map) and off Cananéia on the South Coast of Brazil, State of S. Paulo. The samples were taken from a depth of $10 \mathrm{mts}$, more or less.

Fr. Müller mentioned (1898) the existence of Tornaria which probably belonged to Balanoglossus on the coast of Brazil, but did not describe the larvae. No other scientist has published anything on Brazilian tornariae since.

All larvae studied from the two samples given me, were larvae without tentacles, answering the general description of the larvae belonging to Stiasny-Wijnhoff and Stiasny's type I tornaria (1931). They are generally middle sized to small. The pre-oral area has the form of an anchor in all three of them, they show the general form of a double cone, the ventral 
loop is normal, there are no inferior ventral lobes, lateral lobes are present, with ventral loops, and inferior dorsal lobes, the second and third coeloms are situated next to the gut, the middle-gut being large and swollen, according to Stiasny-Wijnhoff and Stiasny's description (1931, p. 185-186) of the tornariae belonging to type I.

The description of the three different types of tornariae found in the samples did not correspond to the description of any of the already described tornariae belonging to type I of Stiasny-Wijnhoff and Stiasny's, and so new names were given to them - Tornaria mourei n. sp., Tornaria lilianae n. sp., and Tornaria stiasnyi n. sp. Tornaria mourei n. sp. (Pl. I - Figs. 1 to 7 ) Krohn stage $-1,2 \mathrm{~mm}$ height $\mathrm{x} 1,1 \mathrm{~mm}$ wide. The anal plate is protruding, the apical plate is medially or slightly dorsally situated. The ventral belt is neither narrow nor large, taking an almost horizontal position laterally. The pre-oral area is more or less of the same size or slightly smaller than the post-oral area. It is protruding, specially in the region of the upper lip, which is very high. The mouth is highly situated and is small. The second and third coeloms are present in the Krohn stage. There is no anal ring and no secondary circum-anal ciliated band. The circum anal ciliated band is slightly ondulated. The form of the ventral loop (specific character of this tornaria) is very high and narrow, the upper and lower lips of the mouth being parallel (see figs.). The oral area is narrow. The secondary loops are not very projecting; wide at the basis and ending almost pointedly. There are two of them on the internal side of each primary loop and 2-3 on the external side of the same. On the middle loop of the pre-oral and of the post-oral areas there are 3-4 secondary loops. These are usually larger in the middle part of the primary loops and are smaller or less conspicuous at the ends. The median pre-oral and post-oral loops are taller and wider than the lateral corresponding loops (Pl. 1 - Fig. 7). The primary lower dorsal lobes are very deep. They turn a little upwards at the end and are more or less pointed. The secondary lobes, are usually deep, and sometimes narrow opening wide. The lateral lobe is very pronounced, and situated more ventrally than laterally. It has the form of a bottle and ventrally it has a high and large loop, dorsally a smaller but also well defined loop (Pl. I - Fig. 2-7). There are apparently no branchial openings in the Krohn stage. The middle gut is cylindrical in shape and swollen. The hydrocoelom is well developed in the Krohn stage showing muscular thickenings of its walls. The proboscis vesicle or "heart" (pericardium) is present between the canal of the hydrocoelom and the blood vesicle of the proboscis which is beginning its development. The hydrocoelom is large and is pear-shaped or spindle-shaped when laterally looked at. Figs. 4 and 6 (Pl. I) show the same tornaria in the Spengel stage of retrogressive development. It is less transparent then, and shows an annular constriction in the middle of the body, this being club-shaped.

This tornaria differs from the others belonging to type I of StiasnyWijnhoff and Stiasny (the tornaria of Balanoglossus clavigerus, Tornaria bournei, mülleri, krohni, meeki, mielcki, weldoni, ritteri, tergestina) because 
of differences in size, in the shape of the upper and lower lip of the oral area, non existence of the anal ring, the shape of the ventral loop, of the secondary loops, their number, the shape of the middle and hind gut the position of the apical plate, and the pre-oral area and the early development of the coeloms, the shape of the lateral lobe, the position and shape of the mouth, the general shape of the tornaria, etc.

Tornaria litianae n. sp., (Pl. II - Figs. 24-30). Krohn stage-height : $0,7 \mathrm{~mm} \times 0,6 \mathrm{~mm}$ wide. Shape : double cone, like preceding one. Anal plate very little protruding, the ventral belt being almost vertical in position all around the body of the larva, the apical plate is medially situated, or slightly dorsally, the ventral belt is narrow, the pre-oral area is anchor shaped, and is more or less as large as the post-oral area, it is protruding, wide, occupying more or less half of the ventral region of the tornaria, the cœloma are situated next to the intestine. There is an anal ring. There is a non ondulated circum-anal ciliated band and a secondary circum-anal ciliated band. This is a small tornaria, the shape of the ventral loop being more flat than all and it is medially wide, the hollow of the mouth entrance is narrow, and situated in the middle of the body of the larva, the oesophagus therefore being longer than in the other two described tornarix, the oral area is narrow, and small, the upper and lower lips being parallel. The secondary loops are very little projecting and show in number of two to four on the primary loops. The primary lower dorsal lobes are deep and finish near one to the other, following an horizontal course. The secondary lobes are not at all deep. The lateral lobe is large and flat, with a small loop on the ventral side and hardly a loop on the dorsal side. The second and third cœlomic cavities are present. The pore of the hydrocœlom opens a little to the left. The hydrocœlom is pear-shaped when laterally observed and round when seen ventrally. It shows no spores. The muscular band which unites it with the apical plate is long and narrow. The water canal from the hydrocœlom is not distinctly separated from it and is short. The position of the hydrocœlom is between the œsophagus and the stomach on both of which it rests. There is no proboscis vesicle or "heart" (pericardium), nor gill slits or notochord present in the Krohn stage of this tornaria. It is a tornaria very slightly transparent. The long œesophagus is well separated from the stomach which shows a median constriction, the hind-gut being also well separated from the middle gut and having the shape of an inverted short cone. The third cœlom touches with its inferior border the largest part of the inferior middle gut. Both second and third cœloms are lamelliform, and wider than the cœloms of T. stiasnyi and T. mourei. The second cœlom is very near but does not touch the stomach or middle gut.

This tornaria differs from the other described tornaria of type I because of the size, which is very small, the shape of the middle gut, the horizontal lower primary dorsal lobes, the flat secondary loops and lobes, the flat lateral lobe, the existence of both cœloms in the Krohn stage, the non ondulated circum-anal ciliated band, the shape of the ventral loop, etc. 
Plate II - Figs. 29 and 30 show different aspects of a younger stage of probably this same tornaria, captured off the coast of Cananéia, while the Krohn stage was captured farther away off the Island of Bom Abrigo, at another time of the year. The younger larvae are in the young Metchnikoff stage of progressive development. Their identification with the older stage just described, can be done by the shapes of the apical plate, of the middle gut, of the hind gut; the long œsophagus, the vertical position of the ventral belt, the flat anal plate, the anal ring, the small transparency, the flat and large ventral loop, the parallel lips of the mouth opening.

This description was based on one specimen in the Krohn stage and two specimens in the young Metchnikoff stage.

Tornaria stiasnyi n. sp. - Found with greatest frequency (12 specimens from one towing net) in the plankton collected off Cananéia in September 1949. This larva was not caught in the Krohn stage, only in the young Metchnikoff stage, transition stage from Heider to Metchnikoff, older Metchnikoff stage, Spengel and Agassiz stage. The stages of progressive development (Pl. II - Figs. 14 to 21) were caught off Bom Abrigo Island in January, the stages of retrogressive development were caught off Cananéia (Pl. I-II - Figs. 8-13) in September. This larva must therefore belong to an animal with two periods of sexual maturity during one year probably. Although the Krohn stage was not caught it was possible to characterize the larva as being a new kind of type I larva (or of type V) because of its very peculiar anal area, its excentric anus, the apical plate very dorsally situated, the ventral belt being narrow and occupying a vertical position dorsally whereas ventrally in the young stages it is even slightly bent towards the outside of the larva and in elder stages it is horizontal ventrally. The oral area is pushed upwards the apical plate, excepting in the very young stages. The opening is also very elevated, even in the young stages, the whole gut being pushed analwards. There is an anal ring which follows the general form of the anal plate, very much pushed upwards ventrally, and very short dorsally. The ventral loop is extremely elevated and narrow, the dorsal lip of the mouth entrance being parallel to it. Very characteristic are also the long cilia which develop from the circum-anal ciliated band and the small patches of rugose skin which appear all along this band, around the anus, and in the form of a large blotch at the upper and lower turns of the lateral loops in the pre-oral and post-oral bands. There are two small blotches on the anal area dorsally to the anus which can be observed in the Metchnikoff stage already. The oral area is very wide, and so is the lateral lobe, limited by a very large ventral loop and a smaller dorsal loop. The œsophagus, middle and hind gut are well separated from each other, the œsophagus being short, the middle gut cylindrical or oval in the young forms, and the hind gut being in the form of an inverted cone. The cœloms of the collar and trunk are sausage shaped and one is well separated from the other. The trunk cœlom or third cœlom has a pointed end and is larger than the collar or second cœlom. They are both present in the elder stages. These showed a 
great variation in size between $1,1 \mathrm{~mm}$ in height $\mathrm{x} 0,85 \mathrm{~mm}$ in width, and $0,85 \mathrm{~mm}$ in height $\mathrm{x} 0,59 \mathrm{~mm}$ in width. The Krohn stage having not been captured, it is probable that the larva may attain a larger size than the largest indicated above. The hydrocœlom is pear shaped and shows a very long muscular band of attachment to apical plate in the young forms. The elder forms show a short muscular band, well delimited from the hydrocœlom. The water pore is situated more to the left, and the canal which unites it to the hydrocœlom is very thin and well delimited from the hydrocœlom from the Metchnikoff stage onwards. The heart, proboscis vesicle or pericardium is present in the Spengel stage, as well as the bunch of cells which will form the proboscis blood vessel. Two branchial openings are present in the Spengel stage. This larva does not look like any of the other larvae of the type I of Stiasny-Wijnhoff and Stiasny. It looks very much like Tornaria hubbardi Ritter \& Davis 1904, with which it has several features in common, but it differs from that tornaria by the fact that it shows a smaller number of gill slits, a well delimited apical plate, a more protruding anal area, a smaller oral area, a lateral lobe, larger pre-oral and post-oral bands. The general form of the larva is not a pointed bell shape, but a rounded bell shape. T. hubbardi as well as $T$. stiasnyi are being compared in the Agassiz or Spengel stage. According to Stiasny, T. hubbardi was described in the Agassiz stage of development, but Stiasny had only that stage for description. The tornaria under description, when considered apart from the younger stages, would also give the impression of an Agassiz stage of development, because of the out jutting coneshaped area, the gut pushed towards the anal region, the very large hydrocœlom, the longitudinal ciliated band being pushed towards the apical plate, but, when compared to the younger stages, it will be observed that already in the transitional stage between Heider and Metchnikoff stages, the larva shows all the above mentioned characteristics excepting the large hydrocœlom and perhaps the outjutting coneshaped anal area. This last feature does not seem so important even for Stiasny himself, because that author could not have observed it in $T$. hubbardi, whose anal area is flat. I should place both T. hubbardi and T. stiasnyi (Pl. I - Fig. 8) in the Spengel stage and consider Pl. II Fig. 13 of Tornaria stiasnyi as being in the Agassiz stage. The reasons for this consideration are that in Pl. I - Fig. 8 the tornaria still shows the rests of secondary loops and lobes and is still very transparent, the whole larva having stretched a bit and diminished in size. The cœloms are larger - they have grown. Fig. 10 shows a larva typically in Spengel stage, even the circular constriction in the middle and the clavate form of the larva can be observed. The œsophagus wall is darker because of the muscular growth of its walls, in Pl. II - Fig. 13 which shows an Agassiz stage of retrogressive development.

Types of these three new tornarix are kept at the Museum of the Instituto Oceanográfico, in São Paulo. Topotypes are in the Museu Paranaense, in Curitiba. 
As to establishing the correspondence between these larvae and the adult animals, it is necessary to remember the distribution of the Enteropneusta in the South of Brazil (see map). In the State of Rio de Janeiro (Spengel 1893, p. 126) Glossobalanus minutus (Kowalewsky), Balanoglossus apertus (Spengel), and Schizocardium brasiliense Spengel occur. This last species also occurs in S. Sebastião in the State of S. Paulo, as well as Balanoglossus gigas Fr. Müller (Sawaya 1951, p. 730). A smaller Balanoglossus also occurs in Santos in the State of S. Paulo. In Paraná, on the Caiobá Beach we find Balanoglossus paranaensis Björnberg and a similar Balanoglossus in the Ilha do Mel. In Santa Catarina, another state further south, a small Balanoglossus and a large one B. gigas occur near to Florianópolis.

Of the places mentioned above, Caiobá is the nearest to Cananéia, where the tornariz were collected, one of them therefore probably belonging to $B$. paranaensis.

It is the opinion of Mr. \& Mrs. Stiasny (1931, p. 185) that T. hubbardi, which is very similar to $T$. sliasnyi should belong to a Vth type of tornaria, with different characteristics from the Ist, IInd, IIrd and IVth types. The Stiasnys also wrote that this T. hubbardi and therefore the Vth type of larvae might belong to Schizocardium, whose larva is not known. If that should be true T. stiasnyi would probably belong to Schizocardium brasiliense. It is necessary to rear the larva and thus solve the problem.

\section{BIBLIOGRAFIA}

Agassiz, A.

1872. Tornaria, the young stage of Balanoglossus. The Ame r. Nat. VI, p. 636-637. BALFotrR, F. M.

1880. Larval forms, their nature, origin and affinities. Quart. Journ. Micr. Sci. Vol. XX, p. 381-407.

Bateson, W.

1884. The early stages in the development of Balanoglossus (Sp. incert.). Quart. Journ. Micr. Sci. Vol. 24, p. 207-236.

1884a. Note on the later stages in the development of Balanoglossus kowalews kyi (Agassiz), and on the affinities of the Enteropneusta. Proc. Roy. Soc. London, Vol. 235, p. 23-30.

1885. Further stages in the development of Balanoglossus kowalewskyi, with a suggestion as to the affinities of the Enteropneusta. Quart. Journ. Micr. Scí. (N.S.). Vol. 25, p. 81-122 (Supplement).

Bourne, G. C.

1889. On a tornaria found in British seas. Journ. Mar. Biol. Ass. Vol. 1, p. 63-68. BURdon-Jones, G.

1951. Observations on the spawning behaviour of Saccoglossus horsti Branmbell \& Goodhart, and of other Enteropneusta, Journ. Mar. Biol. Ass. Vol. 29, p. $625-638$.

Cunningham, J. T.

1886. Tornaria and Actinotrocha in the British Coasts. Nature. Vol. 34, p. 361. DAWYDOFF, C.

1940. Classe des Entéropneustes, in Traité de Zoologie, Tome XI, de P. Grassé, p. 432-442.

1940a. Quelques observations sur le développement embryonnaire d'un Balanoglosse de l'Indochine. Compt. Rend. Acad. Sci. Paris. Vol. 221, fasc. 16, p. 332-334. 
Horst, C. J. van der

1936. Planctosphaera and Tornaria. Quart. Journ. Micr. Sci. Vol. 78, fasc. 4, p. 605-613.

KIRK, H. B.

1937. The embriology of Dolichoglossus otagœensis Benham (Abstract). Report of the 23rd meeting of the Austr. and N. Zeal. Assoc. Adv. Sc., 137-138.

MÜLLER, Fr.

1898. Observações sôbre a fauna marinha de Santa Catarina. Rev. Mus. Paulista, Vol. III, p. 33-40.

Sawaya, P.

1950. Reencontro do B. gias Fr. Müller no litoral brasileiro. Bol. Inst. Paulista de Oceanografia. Vol. I, fasc. 1, p. 135-138.

1951. Balanoglossus gigas rediscovered on the Brazilian coast. Nature. Vol. 167, p. 730.

Spengel, J. W.

1893. Die Enteropneusten des Golfen von Neapel. Fauna u. Flora des Golfes von Neapel. Mon. 18, 758 p., 37 pl.

Stısny, G.

1914. Studien über die Entwicklung des Balanoglossus clavigerus. Delle Chiaje. Mitt. Zool. Station Neapel. Vol. 22, p. 255-290.

1921. Die Tornarien-Sammlung von Dr. Mortensen's Pacific Exped. 1914-1916-VII. Videns. Medd. fra Dansk Nat. For. Kjöbenhavn. Vol. 73, p. 123-137.

1921a. Eine neue Tornaria aus dem ostindischen Archipel (T. sunieri) Zool. Meded. Leiden. Vol. 6, p. 101-107.

1921b. Die Westindischen Tornarien. Versl. gewone Vergader. wiss. Natur. Afdeel., 29 , p. $220-253$.

1925. Die Tornaria von Helgoland. Zeit. f. wiss. Zool. Vol. 125, p. 435-447.

1926. Zwei neue Tornarien aus der Nord-See. Zool. Anz. Vol. 66, p. 149-165.

1927. Eine neue Tornaria aus der Adria. Zool. Meded. v. Rijsk. Mus. v. Naturhist. Leiden. Deel XI, p. 37-45.

1928. Die Tornarien Sammlung von Japan. Zool. Jahrb. Abt. Syst. Vol. 56, p. 67-92.

1934. Ueber einige exotische Tornarien. Verh. K. Akad. Wetemsch. Amst. Proc. Sect. Sci. Vol. 38, fasc. 2, p. 1026-1036.

1935. Die Tornarien der Snellius Exped. Konik. Akad. van Wet. Amst. proc. Sect. Scien., 37-6-10, p. 1020-1037.

Stiasny-Wijnhoff, G. \& Stiasny, G.

1931. Die Tornarien. Kritik der Beschribung und Vergleich saemtlicher bekannter Enteropneusten larven. Erg. Fortsch. d. Zool. Vol. 7 (1/2), p. 38-208.

Токіока, Т.

1937. Tornaria susakiensis n. sp., a new tentaculated Tornaria-larva. Annot.

Zool. Jap. Vol. 16, p. 341-344.

\section{EXPLICAÇÃO DAS FIGS. DA ESTAMPA I}

Fig. 1 - Aspecto de frente de Tornaria mourei n. sp., vista ventral, no estádio Krohn, mostrando área pré-oral, com linguetas e lobos secundários, lingueta ventral, anel ciliado circular inferior, boca, estômago com celomas apostos e intestino final, anus e área anal (Tamanho: $1,24 \mathrm{~mm} \mathrm{x} \mathrm{1,29} \mathrm{mm}$ ).

Fig. 2 - A mesm a tornária desenhada de lado e vista um pouco de cima para mostrar o lobo la teral, o esôfago, o hidrocela, o pericárdio e canal de escoamento com hidróporo.

Fig. 3 - A mesma tornária da Fig. 1, vista de lado, (dorsal e ventral) para mostrar os celomas lateralmente e a área anal.

Fig. 4 - Tornaria mourei n. sp., em estado Spengel, vista dorsalmente, mostrando a placa apical, por transparência, o hidrocela, o pericárdio, o hidróporo, o 
intestino médio e final, os celomas e involução das linguetas e lobos secundários (Tamanho: $1,1 \mathrm{~mm} \times 0,8 \mathrm{~mm}$ ).

Fig. 5-A mesma tornária da Fig. 4, vista lateralmente.

Fig. 6 - A mesma tornária da Fig. 4, vista parcialmente de lado, ventralmente e de cima.

Fig. 7 - Aspecto planificado das faixas ciliadas de Tornaria mourei n. sp., no estádio Krohn, com todas as linguetas e lobos secundários (desenho composto das figuras 1,2 e 3 ).

Fig. 8 - Estádio Spengel da Tornaria stiasnyi n. sp., vista lateralmente e do conjunto hidrocela, pericárdio, canal de escoamento, vistos do outro lado da mesma tornária, em detalhe (Tamanho da tornária: $1,0 \mathrm{~mm} \times 0,8 \mathrm{~mm}$ ).

Fig. 9 - A mesma tornária da Fig. 8, vista de cima.

Fig. 10 - A mesma tornária da Fig. 8, vista ventralmente.

\section{EXPLICAÇÃO DAS FIGS. DA ESTAMPA II}

Fig. 11 - A mesma tornária da Fig. 8, vista dorsalmente.

Fig. 12 - Tornaria stiasnyi n. sp., entre estádio Spengel e Agassiz, vista de lado e de dorso (Tamanho : $0,6 \mathrm{~mm} \times 0,92 \mathrm{~mm}$ ).

Fig. 13 - T. stiasnyi n. sp., no estádio Agassiz, vista lateralmente e de dorso (Tamanho : $0,9 \mathrm{~mm})$.

Fig. $14-$ T. stiasnyi n. sp., em estádio Metchnikoff adiantado (Tamanho : 0,56 mm x $0,30 \mathrm{~mm})$.

Fig. 15 - T. stiasnyi no estádio Heider a Metchnikoff de desenvolvimento progressivo, vista ventralmente (Tamanho: $0,37 \mathrm{~mm} \times 0,30 \mathrm{~mm}$ ).

Fig. 16 - A mesma tornária da Fig. 15, vista lateralmente.

Fig. 17 - A mesma tornária da Fig. 15, vista dorsalmente.

Fig. 18 - T. stiasnyi, em estádio de desenvolvimento imediatamente seguinte ao da tornária da Fig. 15 (vista lateral ; tamanho: $0,51 \mathrm{~mm} \times 0,65 \mathrm{~mm}$ ).

Fig. $19-T$. stiasnyi, em estádio Metchnikoff jovem de desenvolvimento, seguinte ao da tornária da Fig. 18, vista dorsalmente (Tamanho : $0,46 \mathrm{~mm} \times 0,65 \mathrm{~mm}$ ).

Fig. 20 - Tornária da Fig. 19, vista ventralmente.

Fig. $21-T$. stiasnyi, no estádio Metchnikoff jovem, vista lateralmente (Tamanho $0,51 \mathrm{~mm} \times 0,35 \mathrm{~mm})$.

Fig. 22 - Hidrocela e cordão muscular de T. stiasnyi, em estádio Spengel de desenvolvimento, vista de cima.

Fig. 23 - Hidrocela, pericárdio, futuro seio sanguíneo, canal de escoamento e hidróporo de T. stiasnyi, estádio Spengel de desenvolvimento regressivo, vista lateralmente.

Fig. $24-T$. lilianæ n. sp., no estádio Krohn de desenvolvimento, vista dorsalmente (Tamanho : $0,72 \mathrm{~mm} \times 0,64 \mathrm{~mm}$ ).

Fig. $25-T$. lilianæ n. sp., da Fig. 24, vista lateralmente.

Fig. $26-T$. lilianæ n. sp., da Fig. 24, vista ventral.

Fig. 27 - Aspecto planificado das faixas ciliadas de T. lilianæ n. sp. (desenhado pela composição das Figs. 24, 25, 26 e 28).

Fig. $28-T$. lilianx, vista de cima (apical).

Fig. $29-T$. lilianæ, no estádio Metchnikoff jovem de desenvolvimento, vista lateral. (Tamanho: $0,39 \mathrm{~mm} \times 0,27 \mathrm{~mm}$ ).

Fig. $30-T$. lilianæ, no estádio Metchnikoff jovem de desenvolvimento, vista lateros dorsal. 


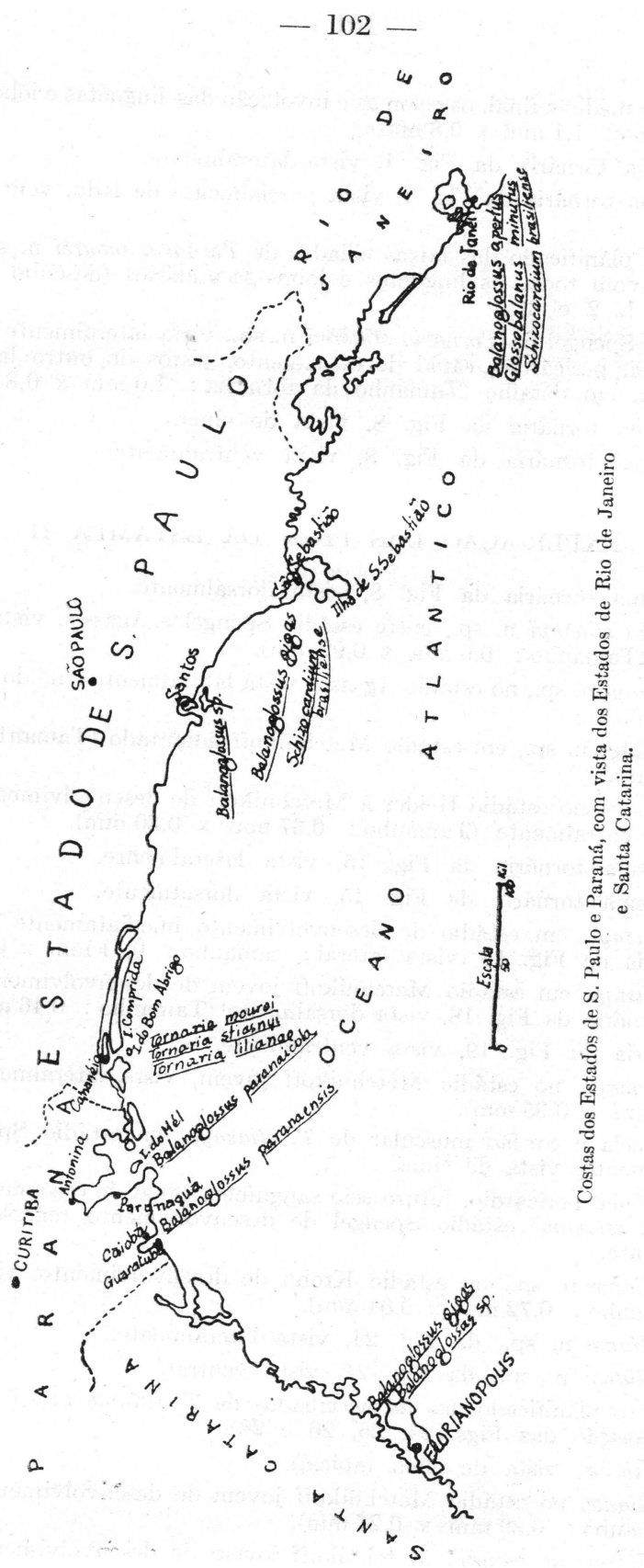



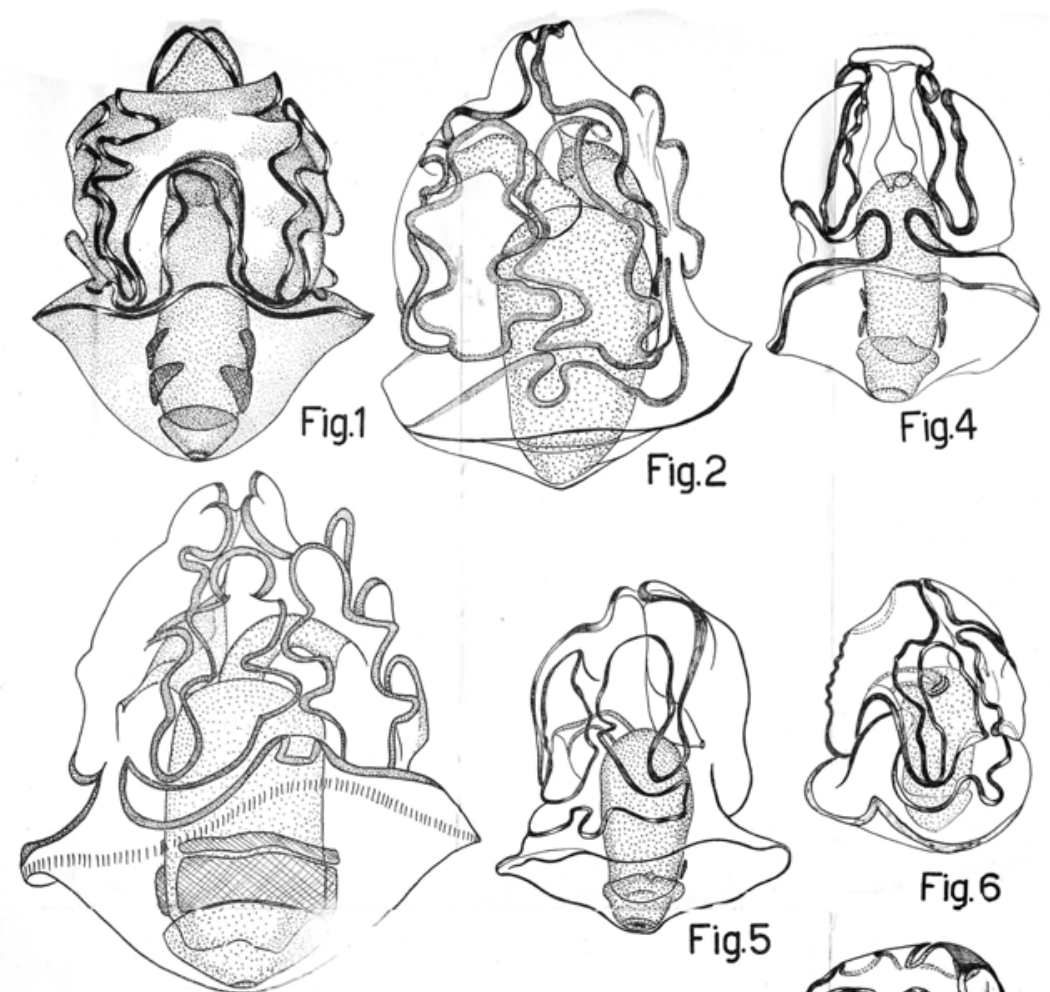

Fig. 6
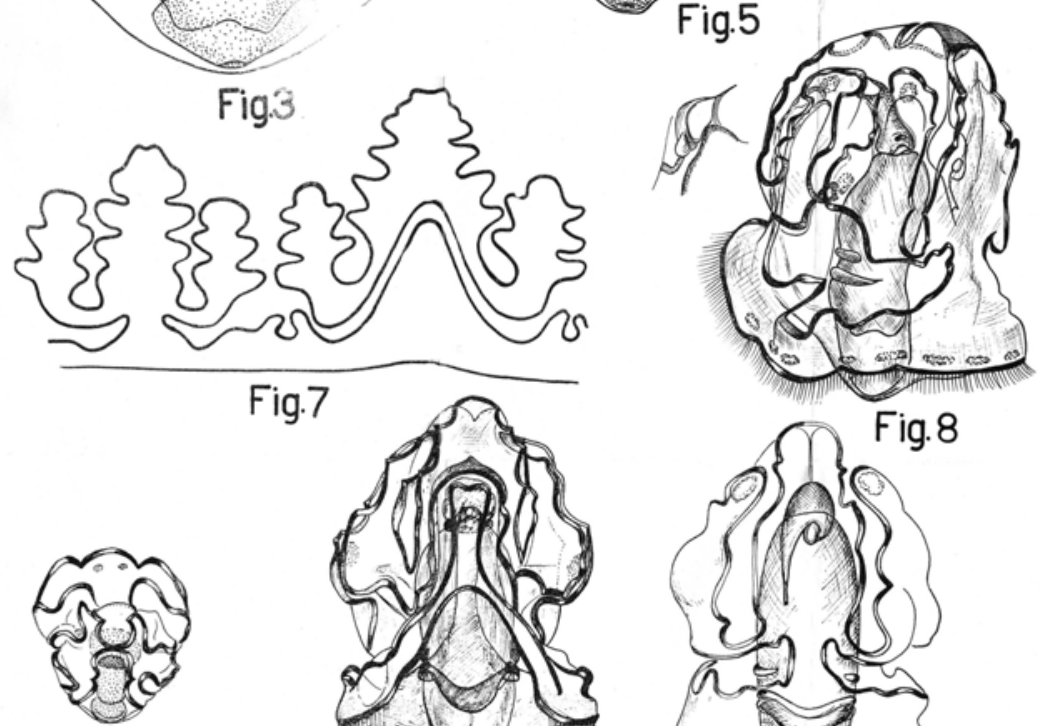

Fig. 3

Fig.9

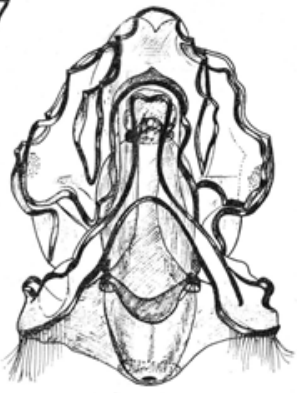

Fig.10

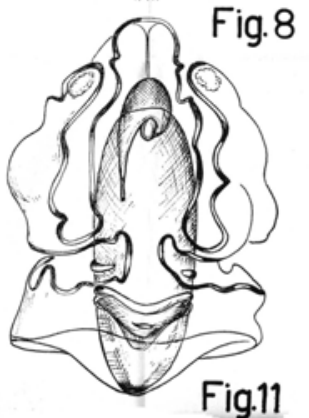




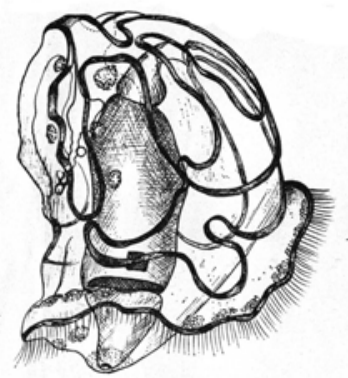

Fig.12

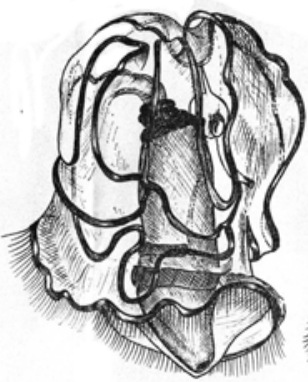

Fig.13
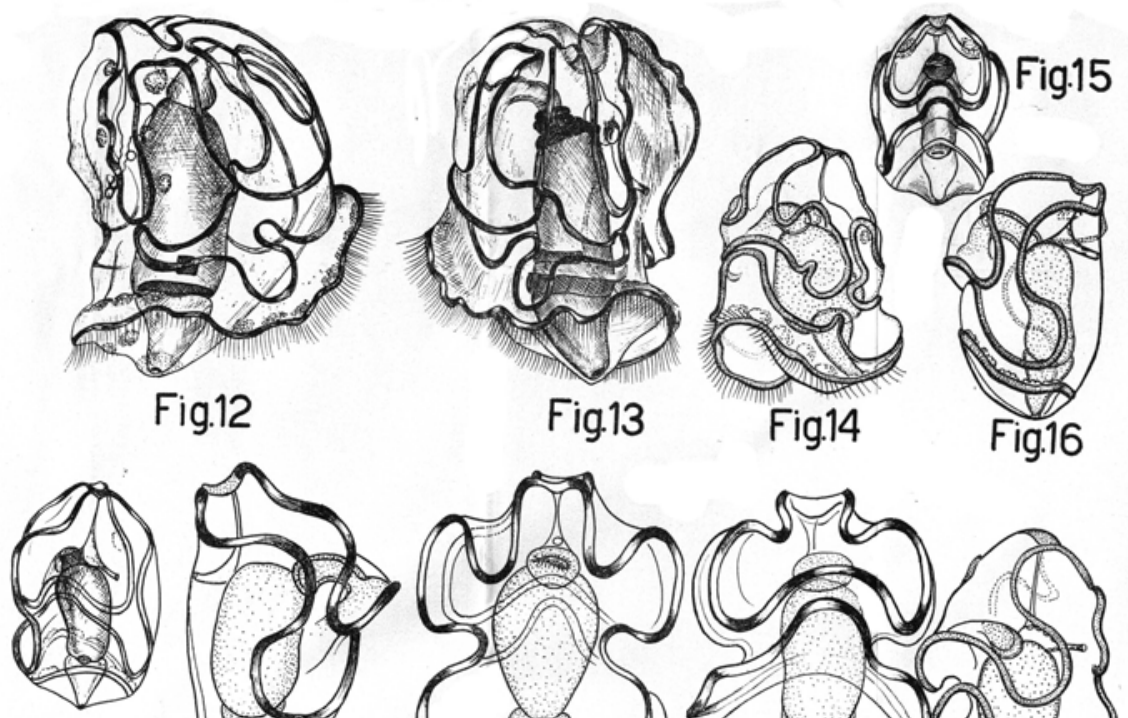

Fig.17
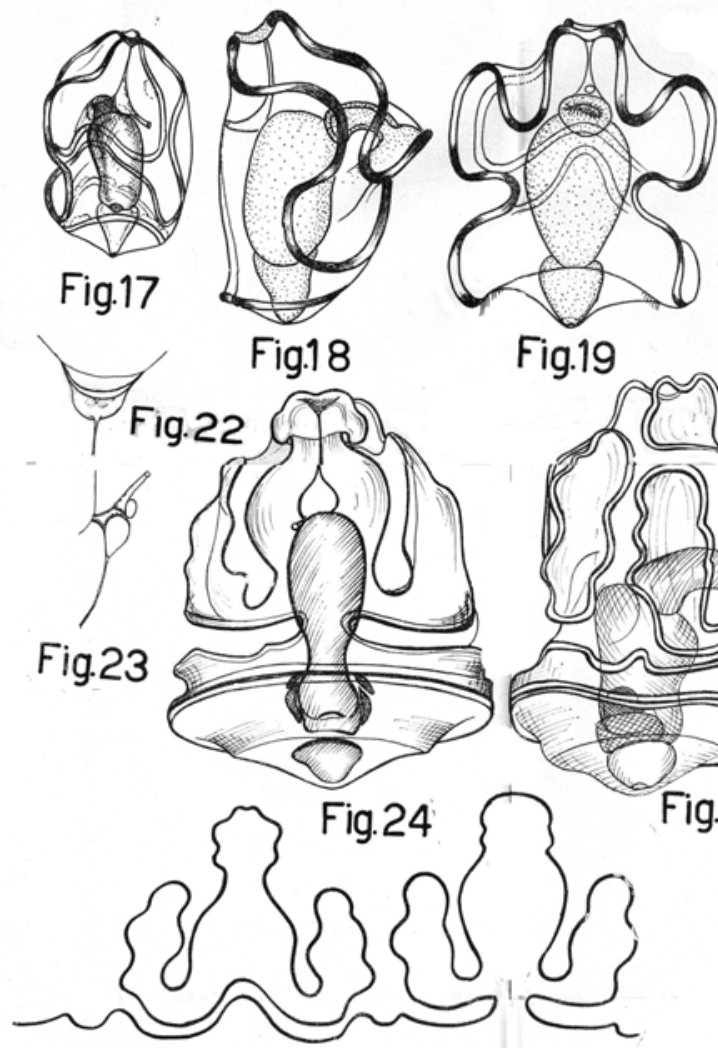

Fig.19

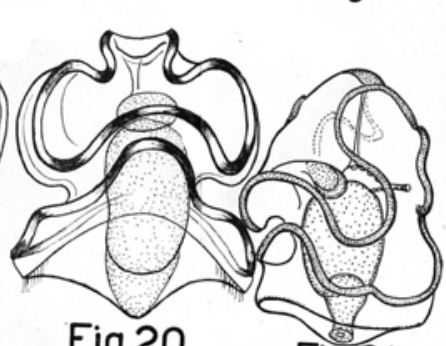

Fig.20

Fig.21
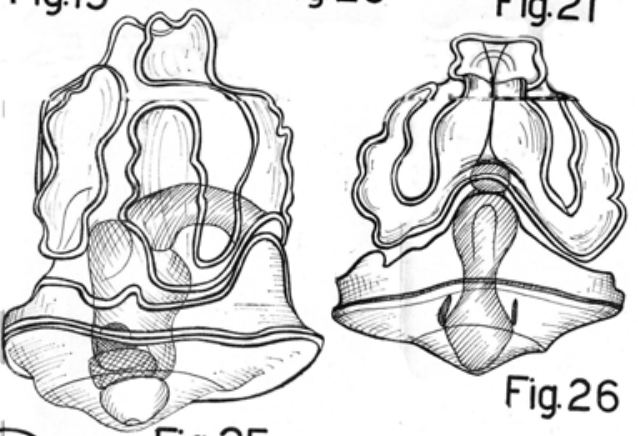

Fig.25

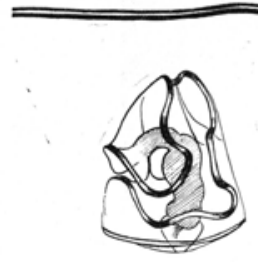

Fig.29
Fig.27

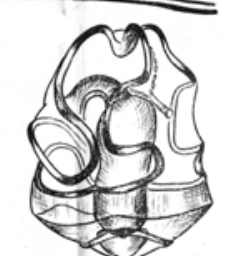

Fiq. 30

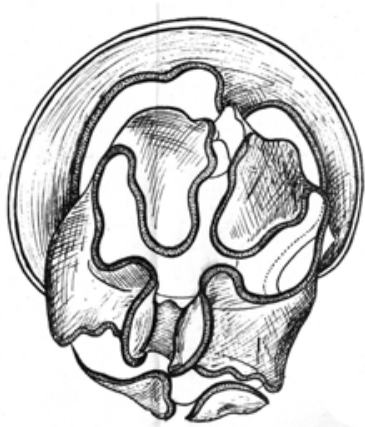

Fig.28 